\title{
Strong exciton-photon coupling in a length tunable optical microcavity with $J$-aggregate dye heterostructures
}

\author{
C. E. Finlayson,, ${ }^{\text {a) }}$ G. Vijaya Prakash, ${ }^{\text {b) }}$ and J. J. Baumberg \\ School of Physics and Astronomy, University of Southampton, Southampton SO17 1BJ, United Kingdom
}

(Received 23 August 2004; accepted 10 November 2004; published online 20 January 2005)

We report the incorporation of thin films of a cyanine dye $J$ aggregate into a versatile, length tunable, optical microcavity. The dense $J$-aggregate layers give an optical response that can be modified by embedding them at specific positions within heterostructures of dielectric and metal layers. The microcavities are composed of separate gold mirrors, which can be individually nanopositioned, and give sharp resonant modes in the red/near-infrared region of the spectrum. With the dye layer favorably placed, anticrossing behavior is observed as the cavity modes are successively swept through the absorption resonance. Large Rabi splittings of up to $170 \mathrm{meV}$ are achieved at room temperature, agreeing well with predictions from a transfer-matrix model. These strongly coupled microcavities pave the way for microelectromechanical systems-integrated microdevices with tailored nonlinear optical properties. (C) 2005 American Institute of Physics. [DOI: $10.1063 / 1.1850189]$

The use of optical microcavities to control the spectral, spatial, and temporal characteristics of spontaneously emitted light currently represents an area of intense scientific interest. ${ }^{1-4}$ In particular, the so called "strong-coupling" regime, when resonant-cavity modes are matched in energy with resonances in the confined medium, such as atomic or molecular transitions, has expanded into a very rich field for the study of fundamental physics and applications, including cavity quantum electrodynamics (QED). Such strongly coupled systems produce anticrossing dispersion behavior (Rabi splitting), as well as admixture quasiparticles or "polaritons," which have been observed to possess drastically different radiative dynamics, carrier scattering properties, and quantum statistics, than the weakly coupled photon and exciton states. ${ }^{6-8}$ This results in modified spontaneous emission that makes microcavities operating in the strongcoupling regime highly attractive for practical semiconductor systems, particularly in developing low-threshold lasers ${ }^{9}$ and resonant-cavity light-emitting diodes (LEDs). ${ }^{10}$ In this letter, we demonstrate strong exciton-photon coupling in a versatile, length tunable, optical microcavity, based on a piezocontrolled, metal-coated, optical fiber. Our techniques have many attractive aspects compared to widely reported monolithic planar microcavity structures, which require wedge or angle tuning for detection. This geometry allows us to directly compare the Rabi splitting for different cavity lengths in the same structure.

A schematic of the microcavities used in the experiments reported in this Letter is shown in Fig. 1. The bottom mirror of the cavity is produced by thermally evaporating $28 \mathrm{~nm}$ of $\mathrm{Au}$ onto an optically flat indium-tin-oxide (ITO) glass substrate. The upper mirror is formed by thermally evaporating a 28 -nm-thick film of Au ( $80 \%$ reflectivity at $\lambda=750 \mathrm{~nm}$ ) onto a perpendicularly cleaved stripped single-mode optical fiber (SM660) of $100-\mu \mathrm{m}$ outer diameter, which is mounted in an $X Y Z$ piezoelectric nanotranslation stage and aligned normally to the bottom mirror film. In order to investigate the transmittance properties of the structures, a broadband white-

\footnotetext{
${ }^{a)}$ Electronic mail: cef@phys.soton.ac.uk

${ }^{b)}$ Present address: Indian Institute of Technology (IIT) Hauz Khas, New Delhi, 110016, India.
}

light source is focused to a $\sim 10 \mu \mathrm{m}$ spot on the rear side of the bottom mirror film. Spectra are recorded by directly coupling the output end of the fiber to a $0.5-\mathrm{m}$ monochromator and a nitrogen-cooled charge-coupled device (CCD) of combined $0.05-\mathrm{nm}$ resolution. All the measurements reported were carried out at room temperature.

These cavities have a tunable longitudinal length between the mirrors, $L$, which can be continuously reduced down to $\sim 1 \mu \mathrm{m}$, with a fine-tuning resolution of a few nanometers. This facilitates the tuning of cavity modes to cover the entire red and near-infrared region of the spectrum (below $\lambda=550 \mathrm{~nm}$, the strongly absorbing nature of Au limits the cavity finesse). This extremely large operating bandwidth contrasts with conventional monolithic $\lambda / 2$ cavities, where off-axis angle tuning is often used to demonstrate the blueshifting of resonant modes across a limited range of photon energies. We are able to achieve $Q$ factors in the range of 10-100, with corresponding cavity finesse of up to 10 . These cavity photon linewidths are sufficient to allow the strongcoupling regime to be observed using high oscillator strength media, such as various organic dyes ${ }^{11}$ and molecular semiconductors. ${ }^{12}$

In our particular experiments, a cyanine dye (Hayashibara NK2751), which exhibits a $J$-aggregating property in a self-ordered, thin-film form, was used as the active medium. This $J$ aggregate is characterized by a narrow, high oscillator

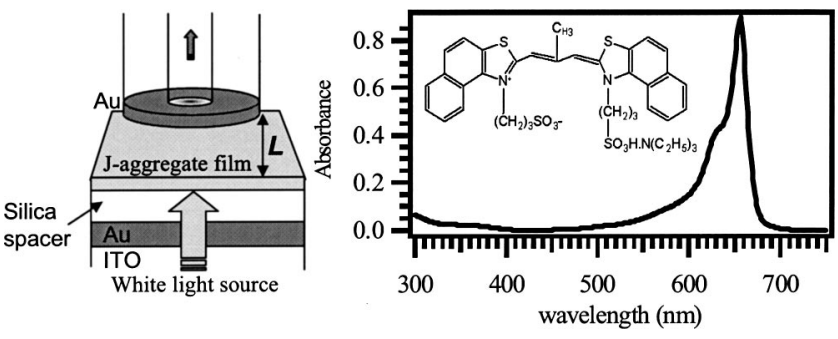

FIG. 1. Schematic of the structure of the length tunable planar microcavities used in our experiments. The optical fiber, onto which the top mirror of the cavity is deposited, is mounted on an $X Y Z$ piezocontroller and this enables fine tuning of the cavity length $L$. Also shown is the absorption spectrum of drop-cast films of the cyanine dye (Hayashibara NK2751), showing a 650 -nm peak from $J$-aggregate formation. The chemical structure of the dye is shown as an inset. 


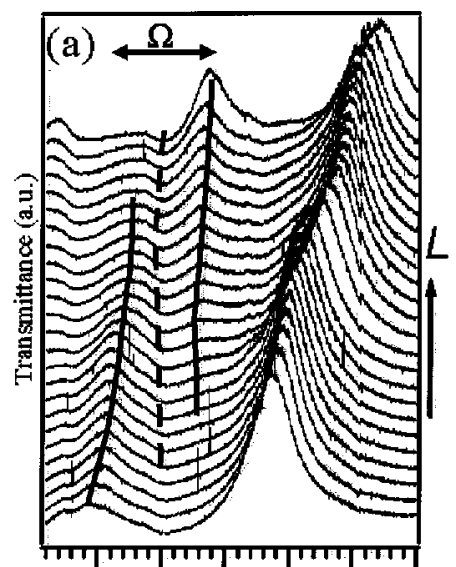

$600 \quad 650700 \quad 750800850$ Wavelength $(\mathrm{nm})$

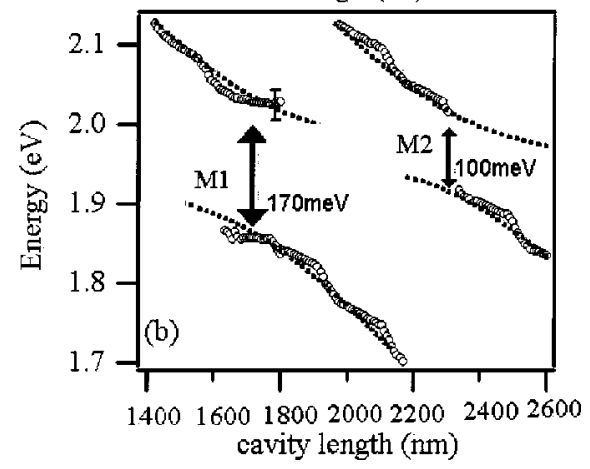

FIG. 2. (a) Broadband transmission spectra as the cavity length, $L$, is tuned. The dispersion of the resonances (solid lines) resolves an anticrossing behavior and Rabi splitting $(\Omega)$ as the cavity modes are tuned across the absorption peak of the dye (dashed line). (b) Extracted peak positions for modes $M 1$ and $M 2$ (open circles). The dashed lines are a guide to eye, constructed using a standard theoretical model (lines).

strength absorption feature at $\lambda=650 \mathrm{~nm}$, as shown in Fig. 1 . Using a convenient drop-casting technique from a dilute solution of the dye in methanol, a uniform thin film is deposited onto the bottom mirror of the cavity. As discussed below, a spacer layer of silica of variable thickness $(0-100 \mathrm{~nm})$ is deposited onto the bottom Au layer, by a technique of radiofrequency sputtering, in order to move the dye away from the expected optical-field node at the mirror. Ellipsometry measurements on films of the dye indicate that they are of thickness of order $10 \mathrm{~nm}$, which probably corresponds to a closepacked arrangement of only a few molecular monolayers.

Here we incorporate the thin layers in resonant microcavities to obtain strong coupling. In Fig. 2(a), the resonant transmitted modes of the dye-containing microcavity are shown, as the cavity is tuned, starting at $L=1440 \mathrm{~nm}$, increasing in steps of $20 \mathrm{~nm}$. As expected, we see the resonant modes of the cavity redshift with increasing cavity length. As the mode $M 1$ is tuned across the dye absorption peak at around $\lambda=650 \mathrm{~nm}$, we see a clear anticrossing behavior characteristic of strong exciton-photon coupling. A similar, although less pronounced, effect is seen as the next longitudinal mode, $M 2$, is tuned through $\lambda=650 \mathrm{~nm}$. The dispersion of these modes showing the split branches of the polariton states is directly shown on an energy versus tuning plot in Fig. 2(b), as the cavity is lengthened. The on-resonance Rabi splittings clearly observed within this range are approximately 170 and $100 \mathrm{meV}$, for the $M 1$ and $M 2$ modes, respectively.
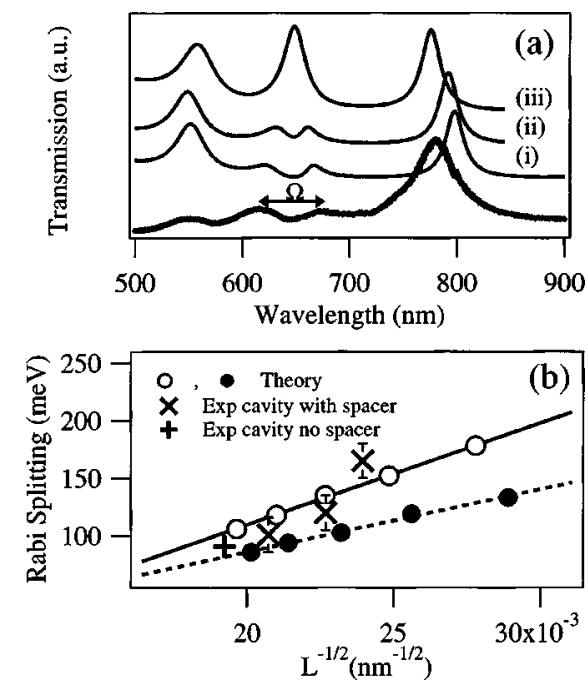

FIG. 3. (a) Transfer-matrix simulations corresponding to $L=1630 \mathrm{~nm}$, (i) with the silica spacer between dye and Au, (ii) in the absence of any spacer, and (iii) with an unfilled cavity. The bold line shows corresponding experimental transmittance data for case (i). (b) Rabi splitting $(\Omega)$ values, derived from our transfer-matrix simulations, plotted against cavity length, as $L^{-1 / 2}$, for cavities with a silica spacer (hollow circles) and without a spacer (solid circles) between the dye and Au layers. The solid line (cavity with spacer) and dashed line (without spacer) are guides to the eye showing the $\Omega$ $\propto L^{-1 / 2}$ relation. Experimental data points for both cavity types are also shown for comparison.

In order to better understand the behavior exhibited by our microcavities, a theoretical model based on a transfermatrix technique, incorporating appropriate optical constants for each of the layers in the system, was developed. In the case of the dye layer, we estimate an extensive oscillator strength of $1 \times 10^{14} \mathrm{~cm}^{-2}$ based on fitting our absorption measurements to a Lorentzian lineshape. The experimental transmission data is compared to the model in Fig. 3(a) for the case of on-resonance Rabi splitting corresponding to a cavity length $L=1630 \mathrm{~nm}$ [(i) in Fig. 3(a)]. The agreement between experiment and theory is satisfactory, and shows that in this geometry the Rabi splitting is of the same order as the longitudinal mode splittings. Also shown in this figure are the theoretical transmission spectra for cavities of equivalent optical path length for the case when the silica spacer is absent [(ii) in Fig. 3(a)] and for an unfilled all-metallic cavity [(iii) in Fig. 3(a)]. This clearly shows the beneficial effect of the spacer to the magnitude of Rabi splitting observed. As can be seen from the intensity profiles in Fig. 4 (plotted at the longer wavelength Rabi-split peak), the coupling to the dye is greatly increased by suitably tailoring both dye position and phase. This differs from bulk organic microcavities in which the oscillator strength is fully distributed across the cavity and not concentrated into a thin quantum well-like layer, which perturbs the optical modes. The magnitude of the strong-coupling effect is well known to depend upon the on-resonance local electric-field strength of the cavity mode within the active medium ${ }^{2}$ and this accounts for the unusually large Rabi splittings we observe compared to previously reported microcavities containing active media of comparable oscillator strength in diluted or matrix-bound form. ${ }^{13}$ We find that the optical field intensity at the dye layer decreases by a factor of about 2 when the 100-nm silica spacer is omitted from the bottom mirror of the cavity, leaving the dye layer on the $\mathrm{Au}$ and closer to a node of the optical field. This, in turn, produces Rabi splittings which are roughly halved.

Downloaded 15 Feb 2005 to 152.78.192.89. Redistribution subject to AIP license or copyright, see http://apl.aip.org/apl/copyright.jsp 

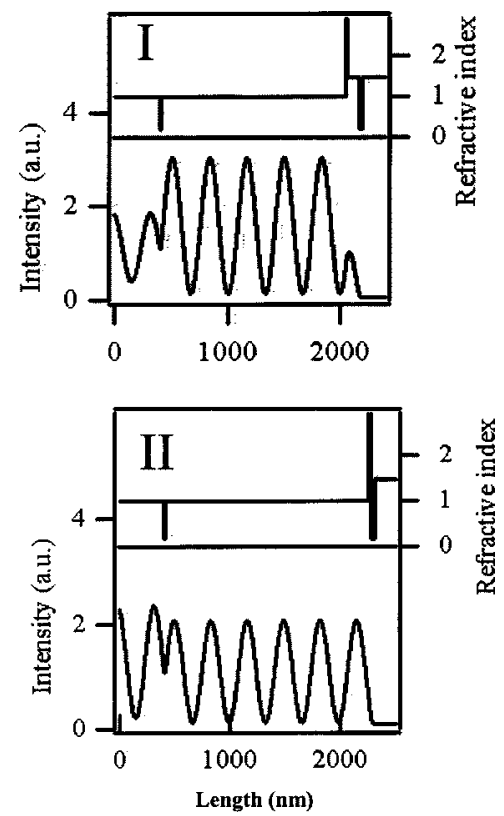

FIG. 4. Spatial optical-field intensity profiles (top mirror at $z=0$ ) corresponding to cases (i) and (ii) from Fig. 3(a), at the longer wavelength transmission peak, $\lambda=660 \mathrm{~nm}$. The refractive index profile shows the position of $\mathrm{Au}$ and dye layers.

The ability to scan the cavity length conveniently allows us to measure anticrossings with successive longitudinal cavity modes. The reduced Rabi splitting of $M 2$ compared to $M 1$ as the cavity is lengthened, as shown in Fig. 2(b), is attributed to the reduced confinement of the optical field due to the longer cavity length. To further emphasize this, the predicted Rabi splittings from our transfer-matrix model for various resonant-cavity lengths are shown in Fig. 3(b). As expected, the model shows that the Rabi splitting is proportional to the inverse square root of the cavity length. ${ }^{2}$ Our experimental data points for the cases of both with and without the silica spacer are found to be in reasonable agreement.

In principle, the strong coupling of photon and exciton ( $J$-aggregate peak) modes can be observed at many different positions of cavity length as the resonant modes are continuously tuned. However, we note that the range of cavity lengths over which we are able to unambiguously observe Rabi splitting (and hence the number of experimental data points that can be extracted) is limited by a number of factors. Firstly, it is not currently possible to tune the cavity to shorter lengths than $\sim 1400 \mathrm{~nm}$, as the geometrical limitations of the present configuration result in reduced cavity stability for such short lengths. Secondly, in order to satisfy the criteria for strong coupling, the optical cavity modes should have sufficient $Q$ factor and finesse, and the bandwidths of both exciton $\left(\Delta \lambda_{\text {exciton }}\right)$ and optical mode $\left(\Delta \lambda_{\text {cavity }}\right)$ should also be closely matched. ${ }^{2,5,14}$ As we increase the length of the experimental cavity beyond $L>3000 \mathrm{~nm}$, the increasing $Q$ factor and finesse become such that the latter of these criteria is no longer satisfied and, hence, Rabi splitting can no longer be clearly observed.

Using our recently developed technology to fabricate tunable confocal microcavities, the bottom mirror may be replaced by a micron-sized, hemispherical Au negativecurvature microreflector. ${ }^{15}$ The advantages of these spherical microcavities, as compared to the planar structures reported in this Letter, are their higher finesse and the increased localization of optical modes, and we predict greatly enhanced strong coupling due to this improved 3D field confinement. Experiments are in progress, using further microfabrication techniques to enhance and separate these sharp, closely spaced, cavity modes using shorter cavities, which will incorporate molecular semiconductors with exciton linewidths which are even narrower than the $J$-aggregate system reported here.

A further advantage of direct length tuning through strongly coupled resonances is the immediate application to study microcavities in which the photons are further confined within the plane, such as photonic pillars ${ }^{16}$ (hence precluding the need for angle or wedge tuning). In terms of prospective applications, these piezocontrolled microcavities are the first step to compatible microelectromechanical systems (MEMS) technology. ${ }^{17,18}$ This will enable the powerful features of photonic state engineering possible in microcavities operating in the strong-coupling regime, ${ }^{19}$ to be combined with integrated fabrication techniques.

In conclusion, we report strong exciton-photon coupling in a length tunable optical microcavity containing a $J$-aggregate dye. Using optimized heterostructures, roomtemperature Rabi splittings as large as $170 \mathrm{meV}$ are observed, tying up well with corresponding theoretical models. The versatility of our tunable microcavity, combined with the convenience of solution processing techniques, provides an attractive platform for the further investigation of strongcoupling light-matter interactions using high oscillator strength materials in optical cavities, and for the future exploitation of these effects in optoelectronic devices.

This work was supported by EPSRC GR/N37261, and GR/S62314. We were greatly aided by useful discussions with David Lidzey and Maurice Skolnick.

${ }^{1}$ H. Yokoyama, Science 256, 66 (1992).

${ }^{2}$ J. G. Rarity, Microcavities \& Photonic Bandgaps, edited by J. G. Rarity and C. Weisbuch (Kluwer Academic, Dordrecht, 1996).

${ }^{3}$ C. E. Finlayson, D. S. Ginger, and N. C. Greenham, Appl. Phys. Lett. 77, $2500(2000)$

${ }^{4}$ K. Vahala, Nature (London) 424, 839 (2003).

${ }^{5}$ C. Weisbuch, M. Nishioka, A. Ishikawa, and Y. Arakawa, Phys. Rev. Lett. 69, 3314 (1992).

${ }^{6}$ P. G. Savvidis, J. J. Baumberg, R. M. Stevenson, M. S. Skolnick, D. M. Whittaker, and J. S. Roberts, Phys. Rev. Lett. 84, 1547 (2000).

${ }^{7}$ P. G. Lagoudakis, M. D. Martin, J. J. Baumberg, A. Qarry, E. Cohen, and L. N. Pfeiffer, Phys. Rev. Lett. 90, 206401 (2003).

${ }^{8}$ D. Porras, C. Ciuti, J. J. Baumberg, and C. Tejedor, Phys. Rev. B 66, 085304 (2002).

${ }^{9}$ P. G. Lagoudakis, M. D. Martin, J. J. Baumberg, G. Malpuech, and A. Kavokin, J. Appl. Phys. 95, 2487 (2004).

${ }^{10}$ N. Takada, T. Kamata, and D. D. C. Bradley, Appl. Phys. Lett. 82, 1812 (2003).

${ }^{11}$ D. G. Lidzey, D. D. C. Bradley, T. Virgili, A. Armitage, M. S. Skolnick, and S. Walker, Phys. Rev. Lett. 82, 3316 (1999).

${ }^{12}$ D. G. Lidzey, D. D. C. Bradley, M. S. Skolnick, T. Virgili, S. Walker, and D. M. Whittaker, Nature (London) 395, 53 (1998).

${ }^{13}$ P. A. Hobson, W. L. Barnes, D. G. Lidzey, G. A. Gehring, D. M. Whittaker, M. S. Skolnick, and S. Walker, Appl. Phys. Lett. 81, 3519 (2002).

${ }^{14}$ Y. Zhu, D. J. Gauthier, S. E. Morin, Q. Wu, H. J. Carmichael, and T. W. Mossberg, Phys. Rev. Lett. 64, 2499 (1990).

${ }^{15}$ G. Vijaya Prakash, L. Besombes, T. Kelf, J. J. Baumberg, P. N. Bartlett, and M. E. Abdelsalam, Opt. Lett. 29, 1500 (2004)

${ }^{16}$ L. C. Andreani, G. Panzarini, and J. M. Gerard, Phys. Rev. B 60, 13276 (1999).

${ }^{17}$ W. Liu and J. J. Talghader, Opt. Lett. 28, 932 (2003).

${ }^{18}$ X. M. Zhang, A. Q. Liu, D. Y. Tang, and C. Lu, Appl. Phys. Lett. 84, 329 (2004).

${ }^{19}$ M. Saba, C. Ciuti, J. Bloch, V. Thierry-Mieg, R. André, L. Si Dang, S. Kundermann, A. Mura, G. Bongiovanni, J. L. Staehli, and B. Deveaud,

Nature (London) 414, 731 (2001). 